\title{
Electrochemical discrimination of dopamine and its metabolites at fluorine-doped tin oxide
}

\author{
Gero Göbel, Fred Lisdat \\ Biosystems Technology, Institute of Applied Life Sciences, Technical University of Applied Sciences, \\ 15745 Wildau, Germany \\ ggoebel@th-wildau.de
}

\begin{abstract}
:
Dopamine and other catecholamines are involved in numerous processes in the neuronal network. Various methods for their analysis have been developed. In order to avoid a disturbance of sensor signal by interfering substances electrochemical sensors for catecholamines apply frequently complex modification strategies. These sensors are characterized often by a rather poor signal stability because of the deposition of polymerization products at the electrode surface. Fluorine-doped tin oxide (FTO) as electrode material allows the direct detection of dopamine via differential pulse voltammetry without expensive modifications and stability problems. This kind of electrode material can discriminate between dopamine and metabolites, is characterized by a wide linear range, shows a high stability of the sensor signal and is not disturbed by typical interfering substances (ascorbic acid, uric acid).
\end{abstract}

Key words: Fluorine-doped tin oxide, FTO, dopamine, L-Dopa, methoxytyramine, interferents

\section{Introduction}

Because of their importance for many signal transduction processes of the human brain and the central and peripheral nervous system there are numerous detection methods for catecholamines. Due to their redox properties electrochemical measurements are predestinated for the analysis.

Screen-printed carbon, carbon paste and glassy carbon electrodes are characterized by a high sensitivity for these neurotransmitters. Hence, these carbon-derived electrodes are frequently used for electrochemical dopamine sensing [1]. In physiological samples the dopamine signal at these electrodes is often superimposed by oxidation currents from dopamine metabolites and interfering substances (ascorbate, ureate) Furthermore polymerization products of oxidized dopamine often lead to a passivation of the electrode surface.

With this respect the electrochemical behavior of dopamine, related metabolites and common interferents is examined at fluorine-doped tin oxide (FTO) based electrodes in the present study.

\section{Results and discussion}

Electrochemical measurements via differential pulse voltammetry (DPV) reveal clear redox signal for dopamine at the FTO electrode at $+320 \mathrm{mV}$ vs. $\mathrm{Ag} / \mathrm{AgCl}$. Compared to SWCNT/carbon electrodes, the current signal of the dopamine precursor L-dopa is reduced significantly and the degradation product methoxytyramine causes nearly no oxidation current (see Fig. 1).

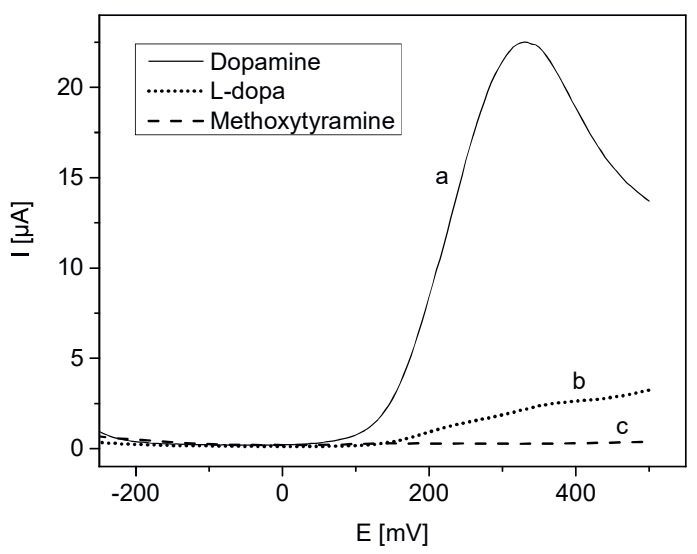

Fig. 1. DPV-measurements of $1 \mathrm{mM}$ dopamine (a), $1 \mathrm{mM}$ L-Dopa (b) and $1 \mathrm{mM}$ methoxytyramine (c) in $20 \mathrm{mM}$ potassium phosphate $(\mathrm{pH}$ 7.2) at FTO electrodes. 
For a sensorial application the dependency of the current response on the analyte concentration as an important parameter has to be considered. At electrodes based on FTO the oxidation current depends linearly on the dopamine concentration in a range from $1 \mu \mathrm{M}$ to $1 \mathrm{mM}$. The $\mathrm{R}^{2}$-value can be determined with 0.999 .

In brain and cerebrospinal liquid the antioxidant ascorbate can be found in rather high concentrations, but also ureate is a frequently found interferent in biological samples. Since FTO allows the distinction between related catecholamine species this electrode material is also examined with respect to these redox active compounds. Even at comparatively high concentrations $(1 \mathrm{mM})$ for both acids nearly no oxidation current at the FTO electrode material can be observed (see Fig. 2). This discrimination can be realized here without the application of additional layers.

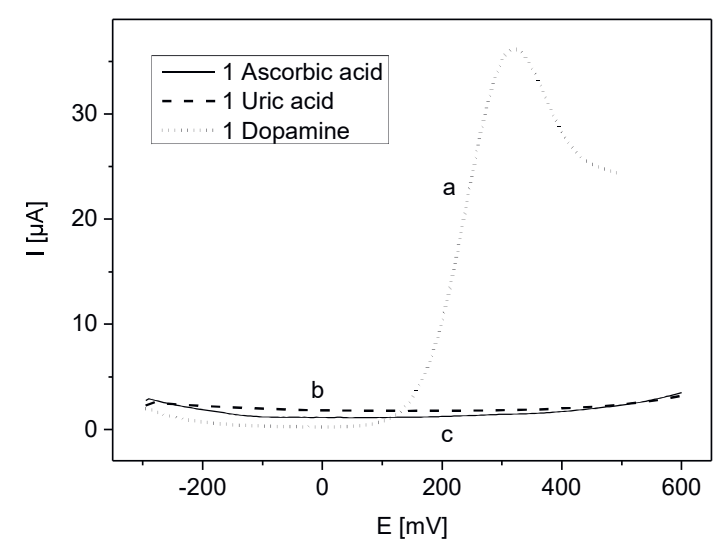

Fig. 2. DPV-measurements of $1 \mathrm{mM}$ dopamine (a), $1 \mathrm{mM}$ ascorbic acid (b) and $1 \mathrm{mM}$ uric acid (c) in $20 \mathrm{mM}$ potassium phosphate $(\mathrm{pH}$ 7.2) at FTO electrodes.

One disadvantage of the electrochemical detection methods for dopamine at numerous electrode materials is the decrease of the current signal during consecutive measurements. In contrast to measurements of dopamine at SWCNT/carbon electrodes via differential pulse voltammetry no decrease of the current signal for a certain dopamine concentration can be observed at a FTO electrode during consecutive DPV measurements.

\section{Conclusions}

FTO as electrode material for the electrochemical determination of dopamine is characterized by a good linearity and high stability of the current signal. It allows the distinction between dopamine and pre-cursors and metabolites as well as the discrimination versus common interferents such as ascorbic or uric acid. Hence, the application of FTO allows not only the construction of reusable dopamine sensors, but also an implementation of dopamine detection in continuously working detection systems.

\section{Ackowledgement}

The financial support by the BMWi (project: $16 \mathrm{KN} 041836$ ) is gratefully acknowledged.

\section{References}

[1] J.A. Ribeiro, P.M.V. Fernandes, C.M. Pereira, F. Silva, Electrochemical sensors and biosensors for determination of catecholamine neurotransmitters: A review, Talanta 160, 653679 (2016); doi: 10.1016/j.talanta.2016.06.066 